\title{
Decentralized Limited-Feedback Multiuser MIMO for Temporally Correlated Channels
}

\author{
Eduardo Zacarías B, Stefan Werner, and Risto Wichman \\ Department of Signal Processing and Acoustics, Aalto University School of Science and Technology, \\ P.O. Box 13000, 00076 Aalto, Finland \\ Correspondence should be addressed to Eduardo Zacarías B, ezacaria@signal.hut.fi
}

Received 9 December 2009; Revised 25 March 2010; Accepted 26 May 2010

Academic Editor: Markku Juntti

Copyright ( $\odot 2010$ Eduardo Zacarías B et al. This is an open access article distributed under the Creative Commons Attribution License, which permits unrestricted use, distribution, and reproduction in any medium, provided the original work is properly cited.

This paper proposes a novel multiuser (MU) multiplexing scheme for temporally correlated multiple-input, multiple-output (MIMO) channels, suitable for systems employing low-rate feedback links. A decentralized solution is obtained by the mobile receivers, which employ interference rejection combiner (IRC) linear filters and command the update of the corresponding peruser antenna transmit weights through compact feedback messages, thus avoiding explicit transmission of channel information. The proposed limited-feedback algorithm outperforms existing MU-MIMO solutions employing quantized matrices, operating at the same feedback overhead. A compensation mechanism is presented, which enables the proposed solution to operate under moderate probabilities of feedback errors, at the expense of a small downlink overhead.

\section{Introduction}

Limited feedback techniques are required in MIMO systems employing frequency division duplex (FDD), because the transmitter cannot directly acquire knowledge of the forward wireless channels of the mobile receivers. These techniques are used to enable different resource management tasks, namely, user scheduling, adaptive modulation, transmit precoding for single user, and transmit precoding for multiuser multiplexing. The work presented in this paper relates to MU-MIMO with linear precoding that is based on limited feedback information.

MU-MIMO systems employing linear precoding have been studied under the assumption of full channel side information (CSI), see, for example, [1,2]. These solutions can be implemented in time division duplex (TDD) systems, where the CSI can be obtained directly from uplink measurements. In FDD systems, however, the feedback links impose a limitation on the amount of CSI that can be obtained by the transmitter, thus motivating the study of limited feedback MU-MIMO techniques. Throughout this paper, we employ the term centralized MU-MIMO solutions to refer to schemes where the multiplexing solution is computed by the transmitter. Although centralized solutions are commonly derived under the assumption of full-CSI, their practical FDD implementations may still employ quantized channel matrices provided by the feedback links, see, for example, $[3,4]$.

This work, in contrast, presents a novel decentralized solution based on the classical interference rejection combiner (IRC) receiver [5] and the limited-feedback eigenbeamforming algorithm [6]. In the proposed scheme, the receivers optimize their respective access point-transmit antenna weights iteratively, to maximize the received signalto-noise ratio in the presence of intracell interference. The feedback messages thus carry weight update commands, as opposed to the channel matrices themselves, and the role of the transmitter is to apply the changes to the corresponding antenna weights. The weight adaptation feedback features a recursion based on unitary coordinate plane transformations, also known as complex-valued Givens rotors. A minimum of one rotor per update is employed, each rotor requiring the feedback of two quantized angles. If allowed by the feedback bit budget, more rotors per update increase the tracking capabilities of the algorithm. The proposed scheme is tailored for the case of single stream transmission per user, 
where each receiver must have more than one antenna in order to be able to use an IRC structure. Furthermore, the performance of the proposed decentralized solution under weight recursions other than [6] is not treated here.

We compare the performance of the proposed algorithm to that of two variants of the (centralized) regularized block diagonalization (RBD) [1], when quantized channel matrices are provided by dominant eigenvector Linde-BuzoGray (DE-LBG) codebooks [4], which have been specifically designed for use with the RBD. Additionally, two recursive channel feedback techniques are also benchmarked. These schemes are based on the limited-feedback eigenbeamforming algorithms D-JAC [6] and ALE-PUB-S [7]. Since the simulation study considers only spatially uncorrelated channels, multiplexing solutions based on channel distribution information (CDI) such as [8] have not been considered.

\section{System Model}

We consider an MU-MIMO system using FDD, where $N_{u}$ users with $N_{r}>1$ antennas each move slowly with respect to a fixed transmitter with $N_{t}$ antennas. The transmission is organized into slots of $L$ symbol periods, each representing the transmission of a different symbol vector $\mathbf{x} \in \mathbb{C}^{N_{u} \times 1}$, where every user is allocated one data stream. The $N_{u}$ users are assumed to experience slowly fading frequency-flat channels, represented by matrices $\mathbf{H}_{i} \in \mathbb{C}^{N_{r} \times N_{t}}, i=1, \ldots, N_{u}$. The transmitter employs a linear precoding matrix $\mathbf{W} \in$ $\mathbb{C}^{N_{t} \times N_{u}}$, with the per-user beamforming vectors $\mathbf{w}_{i} \in \mathbb{C}^{N_{t} \times 1}$ stacked as $\mathbf{W}=\left[\mathbf{w}_{1} \cdots \mathbf{w}_{N_{u}}\right]$.

At the end of the slot, each user can transmit an $n_{b}$ bits feedback message $\mathbf{b}_{i} \in\{0,1\}^{n_{b} \times 1}, i=1, \ldots, N_{u}$, upon which the transmit weights $\mathbf{W}$ are updated. We assume that the feedback channel is delay- and error-free, therefore, the information sent at the end of the slot produces updated weights that are used from the beginning of the next slot. The frequency of the feedback message is related to the slot length and the symbol vector frequency $f_{\mathbf{x}}$ by $f_{\mathbf{b}}=f_{\mathbf{x}} / L, L \gg 1$.

Let $x_{i}(k)$ be the data symbol for user $i$ at symbol period $k$. The received signal for user $i$ reads

$$
\mathbf{y}_{i}(k)=\mathbf{H}_{i}(k) \mathbf{w}_{i}(l) x_{i}(k)+\mathbf{H}_{i}(k) \sum_{m \neq i} \mathbf{w}_{m}(l) x_{m}(k)+\mathbf{n}_{i}(k),
$$

where $\mathbf{n}_{i}(k) \in \mathbb{C}^{N_{r} \times 1}$ is additive circular Gaussian noise such that $\mathrm{E}\left\{\mathbf{n}_{i} \mathbf{n}_{i}^{\dagger}\right\}=\sigma_{i}^{2} \mathbf{I}_{N_{r}}$, and ${ }^{\dagger}$ denotes Hermitian transpose. In (1), $l>0$ denotes the slot index and the update index of $\mathbf{W}$.

Let $\mathbf{Q}_{i} \in \mathbb{C}^{N_{r} \times N_{r}}$ be the covariance of the total disturbance suffered by user $i$, conditioned on the channel matrices $\mathbf{H}_{i}(k)$, the weight vectors $\mathbf{w}_{i}(l)$ and the white noise power $\sigma_{i}^{2}$. This can be written as

$$
\mathbf{Q}_{i}(k, l)=\mathcal{P} \sum_{m \neq i} \mathbf{H}_{i}(k) \mathbf{w}_{m}(l) \mathbf{w}_{m}^{\dagger}(l) \mathbf{H}_{i}^{\dagger}(k)+\sigma_{i}^{2} \mathbf{I}_{N_{r}},
$$

where the same power $\mathcal{P}=\mathrm{E}\left\{\left|x_{i}(k)\right|^{2}\right\}$ is allocated to each user.
The receivers employ linear IRC combiners $\boldsymbol{\omega}_{i}(k, l) \in$ $\mathbb{C}^{N_{r} \times 1}$ based on the receive-diversity combiner [5]. The input to the detector is given by

$$
\begin{gathered}
z_{i}(k)=\boldsymbol{\omega}_{i}^{\dagger}(k, l) \mathbf{y}_{i}(k), \\
\boldsymbol{\omega}_{i}^{\dagger}(k, l)=\frac{\left[\mathbf{H}_{i}(k) \mathbf{w}_{i}(l)\right]^{\dagger} \mathbf{Q}_{i}^{-1}(k, l)}{\mathbf{w}_{i}(l)^{\dagger} \mathbf{H}_{i}(k)^{\dagger} \mathbf{Q}_{i}^{-1}(k, l) \mathbf{H}_{i}(k) \mathbf{w}_{i}(l)},
\end{gathered}
$$

which is implemented at the receiver through a short-term structured estimate $\hat{\mathbf{Q}}_{i}(k, l)$ of $\mathbf{Q}_{i}(k, l)$ defined in (2). It is assumed that the receiver acquires perfect knowledge of $\mathbf{H}_{i}(k)$ from common pilot signals.

\section{Decentralized Closed-Loop MU-MIMO}

This section presents a novel approach to multiuser multiplexing in closed-loop MIMO systems with temporally correlated channels. The mobile users employ the MIMO version of the classical IRC filter [5], as defined in (3). This allows a spatial whitening of the intracell interference produced by the other users. The weight adaptation is based on a decentralized alternating optimization (DAO) procedure. The per-user metric is the received signal-tointerference-plus-noise ratio (SINR).

It can be shown that given $\mathbf{H}_{i}$ and $\mathbf{Q}_{i}$, the combiner (3) produces an SINR

$$
\rho_{i}=\mathbf{w}_{i}^{\dagger}\left[\mathbf{H}_{i}^{\dagger} \mathbf{Q}_{i}^{-1} \mathbf{H}_{i}\right] \mathbf{w}_{i}
$$

Equations (2) and (4) determine how the transmit weight vectors $\mathbf{w}_{i}$ affect the user SINRs, which in turn determine their link-level performance. The DAO approach to maximize the users SINRs would execute the steps.

(1) Given estimates of $\mathbf{H}_{i}, \mathbf{Q}_{i}$, each user optimizes (4) by computing and feeding back the dominant eigenvector of $\mathbf{H}_{i}^{\dagger} \mathbf{Q}_{i}^{-1} \mathbf{H}_{i}$.

(2) The transmitter uses the transmit weights specified by each user, without attempting to jointly optimize the transmit weights. If the weights change, the covariance matrices $\mathbf{Q}_{i}$ change as well, according to (2).

(3) Go to Step 1.

The DAO procedure resembles the alternating optimization (AO) technique [9], but differs in that the DAO does not use a single objective function. This comes from the fact that each receiver has no information about the performance of the others. Therefore, it is difficult to make general claims about convergence, and we resort to static channel simulations to illustrate some convergence properties of the DAO solution. This is given in Section 4.3.

Assuming that the channel coherence time allows for convergence and tracking of the optimal solution, it remains to specify a limited-feedback mechanism to transmit the vectors computed on each iteration, using a small number of feedback bits. This is described next. 
One possibility to implement the DAO for MU-MIMO concept is to use the distributed Jacobi eigenbeamforming (D-JAC) algorithm [6], as means to track the optimal beamforming vectors with limited feedback. The D-JAC algorithm features an update based on unitary coordinate plane transformations, also referred to as complex-valued Givens rotors or Jacobi transformations. This method is designed to track the eigenbasis of a general Hermitian matrix according to its descending-order sorted eigenvalues. It is also possible to track only the dominant eigenvector of the matrix, which makes the method suitable for the proposed DAO. In the following, both transmitter and receiver keep a copy of a unitary matrix $\boldsymbol{\Phi}_{i}$ which contains $\mathbf{w}_{i}$ as the first column, and the update of $\boldsymbol{\Phi}_{i}$ is done synchronously through the feedback message $\mathbf{b}_{i}$. Upon arrival of $\mathbf{b}_{i}(l)$, the transmitter updates $\mathbf{w}_{i}(l)$ by doing

$$
\begin{gathered}
\boldsymbol{\Phi}_{i}(l+1)=\boldsymbol{\Phi}_{i}(l) \mathbf{J}^{1, q}(l), \quad \boldsymbol{\Phi}_{i}(0)=\mathbf{I}_{N_{t}}, \\
\mathbf{w}_{i}(l+1)=\boldsymbol{\Phi}_{i}(l+1) \tilde{\mathbf{w}},
\end{gathered}
$$

where $\mathbf{J}^{1, q}(l)$ is an identity matrix, except for the entries $(1,1)$, $(1, q),(q, 1)$, and $(q, q)$, which contain the nontrivial terms $\cos (\alpha)$ and $\sin (\alpha) e^{j \beta}$ and $\tilde{\mathbf{w}}$ is the first column of the identity matrix of size $N_{t}$. The angle pair $(\alpha, \beta)$ is retrieved from $\mathbf{b}_{i}(l)$ and the coordinate plane $(1, q)$ is selected sequentially with $q \in\left\{2, \ldots, N_{t}\right\}$. Each rotor angle is quantized independently and uniformly, and the ranges for $\alpha$ and $\beta$ are $[0, \pi / 2]$ and $[-\pi, \pi)$, respectively [6]. Note that one can apply (5) more than once per update, provided that the angle pairs for all the rotors are contained in $\mathbf{b}_{i}(l)$. This speeds up the convergence of the D-JAC algorithm, but requires larger $n_{b}$. In the absence of feedback errors, both transmitter and receiver have identical copies of $\boldsymbol{\Phi}_{i}$.

The receivers are responsible for computing the angle pairs for the rotor(s) involved in the update, and for feeding them back to the transmitter. How closely the weights $\mathbf{w}_{i}$ match the dominant eigenvector of $\mathbf{H}_{i}^{\dagger} \mathbf{Q}_{i}^{-1} \mathbf{H}_{i}$ depends on the antenna array sizes $N_{t}, N_{r}$, the feedback message frequency, the channel fading rate and also on how many rotors per update are encoded in $\mathbf{b}_{i}(l)$. When using only one rotor per update, it can be desirable to limit the variations of the transmit weights between updates, to allow for convergence of the D-JAC update. Therefore, instead of tracking the dominant eigenvector of $\mathbf{H}_{i}^{\dagger} \mathbf{Q}_{i}^{-1} \mathbf{H}_{i}$, the algorithm tracks the eigenvector of $\mathbf{H}_{i}^{\dagger} \widetilde{\mathbf{Q}}_{i}^{-1} \mathbf{H}_{i}$, where $\widetilde{\mathbf{Q}}_{i}(l)$ is a low-pass filtered covariance matrix defined as

$$
\begin{gathered}
\widetilde{\mathbf{Q}}_{i}(l)=(1-\epsilon) \widetilde{\mathbf{Q}}_{i}(l-1)+\epsilon \hat{\mathbf{Q}}_{i}(l L-1, l), \\
\widetilde{\mathbf{Q}}_{i}(0)=\mathbf{I}_{N_{r}}, \quad \epsilon \in(0,1],
\end{gathered}
$$

with $\widehat{\mathbf{Q}}(l L-1, l)$ an estimate of $\mathbf{Q}_{i}(l L-1, l)$ defined in $(2)$ and $\epsilon \in(0,1]$ a parameter controlling the filter memory.

The filtered matrix $\widetilde{\mathbf{Q}}_{i}(l)$ is used to compute an auxiliary channel correlation matrix $\mathbf{R}_{i}(l)$, which is the input to the $\mathrm{D}$-JAC algorithm and is defined as

$$
\mathbf{R}_{i}(l)=\mathbf{H}_{i}(l L-1)^{\dagger} \widetilde{\mathbf{Q}}_{i}^{-1}(l) \mathbf{H}_{i}(l L-1),
$$

\begin{tabular}{|c|c|}
\hline U. $i$ : & Set $\mathbf{H}=\mathbf{H}_{i}(l L-1)$, the most recent channel sample \\
\hline U. $i$ : & Set $\hat{\mathbf{Q}}$ to the most recent estimate of $\mathbf{Q}_{i}$ \\
\hline U. $i$ : & $\begin{array}{l}\text { Set } \widetilde{\mathbf{Q}} \text { to the updated filtered covariance matrix } \widetilde{\mathbf{Q}}_{i} \text { from } \\
\text { (6) }\end{array}$ \\
\hline U. $i$ : & compute $\mathbf{R}=\mathbf{H}^{\dagger} \widetilde{\mathbf{Q}}^{-1} \mathbf{H}$ \\
\hline U. $i$ : & $\begin{array}{l}\text { For each rotor in the update: compute the angle pair }(\alpha, \beta) \\
\text { based on } \mathbf{R} \text { and the receive-side auxiliary matrix } \boldsymbol{\Phi}_{i} \text {, as in } \\
\text { [6]. Update } \boldsymbol{\Phi}_{i} \text { and the own weights } \mathbf{w}_{i} \text {. Quantize }(\alpha, \beta) \\
\text { and include it in the feedback message } \mathbf{b}_{i}(l)\end{array}$ \\
\hline Tx.: & $\begin{array}{l}\text { For each rotor in the update of user } i \text { : retrieve }(\alpha, \beta) \text { from } \\
\mathbf{b}_{i}(l) \text {, assemble the corresponding Givens rotor } \mathbf{J}_{i}^{1, q} \text {. } \\
\text { Update the transmit-side } \boldsymbol{\Phi}_{i}, \mathbf{w}_{i} \text { with }(5) \text {. }\end{array}$ \\
\hline
\end{tabular}

TABLE 1: Pseudocode for the proposed algorithm ("DAO-D-JAC"). This process occurs at the end of each slot $l>0$. "U. $i$ " denotes processing at the $i$ th receiver, "Tx." denotes processing at the transmitter.

where the most current channel matrix should be used. In the system model of Section 2, this is the channel at the end of the slot, that is, $k=l L-1$. The matrix $\mathbf{R}_{i}(l)$ and the receive-side version of $\boldsymbol{\Phi}_{i}(l)$ define the update of $\mathbf{w}_{i}(l)$ through one or more Jacobi transformations, as detailed in [6]. For each rotor to be applied in the update, an angle pair is computed, quantized, and included in $\mathbf{b}_{i}(l)$. Furthermore, the computation of the rotor angles guarantees that the first column of $\boldsymbol{\Phi}_{i}$ tracks the dominant eigenvector of $\mathbf{R}_{i}$. This is so because the D-JAC algorithm operates similarly to the Jacobi method for Hermitian matrices [10].

The proposed decentralized MU-MIMO is summarized in Table 1 and referred hereafter to as DAO-D-JAC.

3.1. Compensation of Feedback Errors. In case of feedback errors, the angles $\alpha, \beta$ that define the Givens rotor $\mathbf{J}^{1, q}(l)$ used in the weight update (5) are not the same at the transmit and receive side. This causes that the transmit weights used by the transmitter start to drift from the weights that the receiver is specifying. If uncorrected, this deteriorates the convergence and tracking performance of the proposed solution. To compensate for this effect, two mechanisms are considered.

(1) A simple strategy where the $\boldsymbol{\Phi}$ matrices are reset to identities on both sides, after a fixed number of slots. This prevents accumulation of errors, but affects the tracking performance, since some slots will be required for readjusting the transmit weights. The periodicity of this resetting procedure needs to be adjusted according to the mobile speed and the probability of feedback errors.

(2) A strategy where the received angles are forwarded by the transmitter to each user. The receiver compares the forwarded $\alpha, \beta$ angles with its local values. Upon difference, $\Phi$ reverts to its previous value, and a rotor based on the forwarded angles is applied. In this fashion, the $\Phi$ matrices are synchronized at transmit and receive side, but random perturbations are introduced in the algorithm's convergence, which need to be corrected in subsequent updates. This concept is illustrated in Figure 1. 


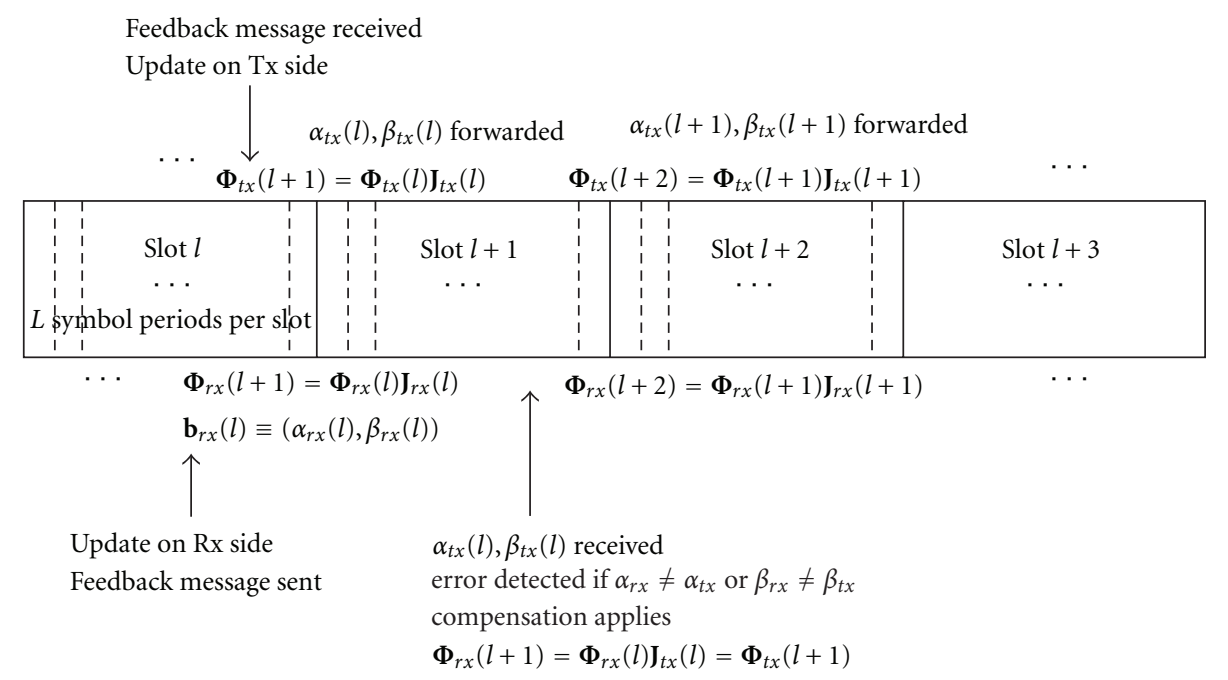

FIGURE 1: Overview of the feedback error compensation through angle forwarding. The transmit weight update is done on both transmit and receive side according to (5), where the rotors $\mathbf{J}$ are built from the angles $(\alpha, \beta)$, which are contained in the feedback message $\mathbf{b}$. The coordinate plane indices $(1, q)$ of the rotors are not shown.

Current standards like the Third Generation Partnership Project (3GPP) Long Term Evolution (LTE) include basic feedback message forwarding as part of the downlink control information (DCI). This comes in the form of the transmit precoding matrix indicator (TPMI), which communicates the receiver which codebook entry has been employed by the transmitter [11]. These bits have channel coding protection, which lowers the probability of undetected errors in the forwarded messages. If the TPMI is to be used with recursive algorithms such as the proposed DAO-D-JAC, then the mechanism (1) can be employed to limit propagation of infrequent errors in the forwarded messages.

A thorough coverage of the compensation of feedback errors is beyond the scope of this paper. Simulations illustrating the performance of mechanism (2) are given in Section 4 under the assumption that no errors occur in the forwarded messages.

\section{Simulations}

We consider a case of $N_{u}=2, N_{t}=4, N_{r}=2$ and a single stream transmission for each user, with 16QAM constellation and a symbol rate of $240 \mathrm{ksymb} / \mathrm{s}$. Both users have the same SNR conditions $\left(\sigma_{1}^{2}=\sigma_{2}^{2}=\sigma^{2}\right)$ and speed relative to the transmitter. The carrier frequency is $2.1 \mathrm{GHz}$, there are $L=160$ symbol periods per slot and the feedback message frequency (once per slot) is $1500 \mathrm{~Hz}$. Rayleigh fading channels are spatially uncorrelated and temporal correlation is generated by a fourth order Butterworth filter adjusted to the maximum Doppler frequency. The feedback overhead is the same for the considered schemes.

The DAO-D-JAC feedback messages $\mathbf{b}_{i}(l)$ contain $n_{b}=6$ bits each, encoding a one-rotor update per user. Each angle is quantized independently and uniformly with three bits, on its respective range. This bit budget per angle gives close to optimal performance for a single-rotor update, in slowly fading channels. The filter parameter $\epsilon$ has been optimized for the mobile speed of $3 \mathrm{~km} / \mathrm{h}$ scenario and set to $\epsilon=0.55$. We assume that each receiver can build a perfect estimate of its covariance matrix $\mathbf{Q}_{i}$ from (2) locally.

4.1. Benchmarking Methods. We compare the performance of the proposed decentralized solution with that of centralized RBD and centralized minimum mean square error (MMSE) solutions. The RBD is chosen, since specific feedback methods have been presented in literature. On the other hand, the MMSE solution serves as a good benchmarking reference under full CSI conditions.

Two types of feedback methods for the centralized MUMIMO are considered: nonrecursive and recursive. All the methods under consideration provide the transmitter with a vectorized and normalized version of $\mathbf{H}_{i}$, namely,

$$
\widetilde{\mathbf{h}}_{i}=\frac{\operatorname{vec}\left(\mathbf{H}_{i}\right)}{\left\|\mathbf{H}_{i}\right\|_{F}} .
$$

Note that this preserves the power relationship between the channel matrix columns, but does not preserve the power relation between channels of different users, as present in the full-CSI scenario. In the following, we summarize briefly the most important details of the benchmarking methods.

4.1.1. RBD. The RBD [1] is a decomposition-based approach, where the precoder matrices $\mathbf{W}_{i}$ are factored as $\mathbf{W}_{i}=\gamma \mathbf{W}_{i, a} \mathbf{W}_{i, b}$. The terms $\mathbf{W}_{i, a}$ separate the users, so that the total multiuser interference (MUI) plus noise at the receivers is minimized. This is done for each user through the singular value decomposition (SVD) of a matrix containing the channel matrices of the other users. Then the terms $\mathbf{W}_{i, b}$ are computed independently for each user, to optimize the transmission through the precoded channel $\mathbf{H}_{i} \mathbf{W}_{i, a}$, for example with eigenbeamforming. Finally, the scalar factor $\gamma$ normalizes the total transmit power and there is no explicit 
control of how much of the total transmit power is allocated to each user. This technique is referred to as "RBD full CSI" in the simulations results. Whenever quantized matrices are used instead of the true channel matrices, the channel feedback method is specified.

The RBD solution tends to null the MUI at high signalto-noise ratios, implying that the IRC filter becomes a matched filter under low noise conditions. A matched filter has been used in the BER simulations. Note, however, that the feedback quantization generates increased MUI and an IRC filter could improve the performance by spatially whitening the MUI. This has not been explored here. Also, the performance of RBD refinements such as the iterative RBD (I-RBD) [1] have been left out.

4.1.2. Total MMSE. The mean square error given channel matrices and noise power can be written in terms of the precoding matrices $\mathbf{W}_{i}$ for each user as

$$
\begin{aligned}
\mathrm{E}\left\{\left\|\mathbf{x}_{i}-\boldsymbol{\Omega}_{i}^{\dagger} \mathbf{y}_{i}\right\|^{2}\right\} \\
\quad=\mathrm{E}\left\{\left\|\left(\mathbf{I}-\boldsymbol{\Omega}_{i}^{\dagger} \mathbf{H}_{i} \mathbf{W}_{i}\right) \mathbf{x}_{i}-\boldsymbol{\Omega}_{i}^{\dagger} \mathbf{n}_{i}^{\prime}\right\|^{2}\right\} \\
\quad=\operatorname{trace}\left[\mathcal{P}\left(\mathbf{I}-\boldsymbol{\Omega}_{i}^{\dagger} \mathbf{H}_{i} \mathbf{W}_{i}\right)\left(\mathbf{I}-\boldsymbol{\Omega}_{i}^{\dagger} \mathbf{H}_{i} \mathbf{W}_{i}\right)^{\dagger}+\boldsymbol{\Omega}_{i}^{\dagger} \mathbf{Q}_{i} \boldsymbol{\Omega}_{i}\right],
\end{aligned}
$$

where $\mathbf{n}_{i}^{\prime}$ is the MUI plus noise term from (1), $\mathbf{Q}_{i}$ is the covariance matrix of $\mathbf{n}_{i}^{\prime}$ from (2), $\boldsymbol{\Omega}_{i}$ is the receive filter matrix from (3) and $\mathcal{P}$ is the average power per symbol per stream. The total MMSE solution for $\mathbf{W}$ can then be found with numerical methods when (3) is used to replace $\boldsymbol{\Omega}_{i}$ and the objective function is the sum of the MSE of each user. This is similar to the "direct optimization" approach of [12], except that we write the MSE explicitly as a function of $\mathbf{W}$, rather than a function of the receive filters $\boldsymbol{\Omega}_{i}$.

4.1.3. Nonrecursive Feedback Methods. The nonrecursive methods are based on static dominant eigenvector LindeBuzo-Gray (DE-LBG) codebooks, which have been presented in the context of limited-feedback RBD [4]. The chosen vector is selected using a minimum chordal distance criterion, and the index is fed back to the transmitter, which reshapes the column vector into the channel matrix estimate. The codebooks are obtained by using the LindeBuzo-Gray algorithm [13]. The chordal distance is used as distortion measure, and the centroids are computed as the dominant eigenvector of the sample covariance matrix of each cluster. The combination of RBD and nonrecursive DELBG codebooks of size $2^{N}$ is referred-to as "RBD-DE-LBG $N$ bits" in the simulations section.

A similar approach for feeding back the optimal beamformer in the MISO case (no vectorizing/reshaping is need) was already considered in [14], where a maximum received SNR criterion is used to partition the training vectors. This is equivalent to the chordal distance criterion in [4]. Another application of the LBG algorithm with eigendecomposition of the cluster covariance matrices can be found in [15], where the goal is to feed back quantized orthogonal bases associated to the channel matrices.

4.1.4. Recursive DE-LBG Codebooks. In general, a given codebook entry can be chosen in two or more consecutive slots, depending on the fading rate and the codebook size. Clearly, these consecutive repetitions of the codebook index do not increase the CSI accuracy at the transmitter, and the associated feedback bits are wasted. For fixed fading rates and vector sizes, it is possible to design hierarchical codebook structures, operating in a nested fashion: a codeword is chosen at the beginning of a period and the following feedback messages indicate codewords from a finer codebook. More details on the design and performance of these nested codebooks can be found in [15]. We implement a similar strategy here, but restrict the codebook hierarchy to two layers and the same codebook sizes on both layers. This is done to keep the feedback rate constant. We use a set of training vectors and first produce one DE-LBG codebook. Then we produce one codebook of the same size for each cluster produced in the previous step. For the particular mobile speed of $3 \mathrm{~km} / \mathrm{h}$, we have chosen periods of ten slots, where the coarse codebook index is followed by nine slots conveying indices within the associated nested codebook. This benchmarking method will be labeled "RBD-2-layer nested DE-LBG" in the simulation results.

Related works in recursive codebooks can be found as switched codebooks [16], which aim to adapt to spatial or temporal channel correlation. A different approach of rotating and scaling a codebook to increase the beamforming accuracy has been explored in the context of spatial correlation in [17].

\subsubsection{Recursive Feedback Based on Eigenbeamforming. Track-} ing the vectorized and normalized channel $\widetilde{\mathbf{h}}_{i}$ defined in (8) is easily related to single-stream eigenbeamforming methods, which signal changes in the dominant eigenvector of the channel correlation matrix $\mathbf{H}_{i}^{\dagger} \mathbf{H}_{i}$. By replacing this matrix with the rank-one matrix $\widetilde{\mathbf{h}}_{i} \tilde{\mathbf{h}}_{i}^{\dagger}$, one can convey $e^{j \phi} \widetilde{\mathbf{h}}_{i}$ to the transmitter, where a phase rotation to the whole vector may appear, depending on the feedback method of choice. This ambiguity, however, does not impact the performance of the RBD and is also inherently present in codebook designs based on the chordal distance.

The first of the eigenbeamforming-based channel feedback methods employs the D-JAC algorithm [6], which is also used to track the dominant eigenvector of $\mathbf{H}_{i}^{\dagger} \mathbf{Q}_{i}^{-1} \mathbf{H}_{i}$ in the proposed decentralized algorithm. Here, however, the Givens or Jacobi rotors operate on $N_{t} N_{r}-1$ planes for the vectorized channel, as opposed to the DAO-D-JAC, where only $N_{t}-1$ planes are required. We refer to this method as "RBD-EBF-CHFB ( D-JAC )" in the simulation results. Only one rotor per update is employed, with $n_{b}=6$.

The second method is based on a partial update (PU) eigenbeamforming scheme. The alignment-enhanced partial update beamforming (ALE-PUB) algorithm [7] computes and signals the PU of the optimal beamforming vector as to maximize the received maximum ratio combining 


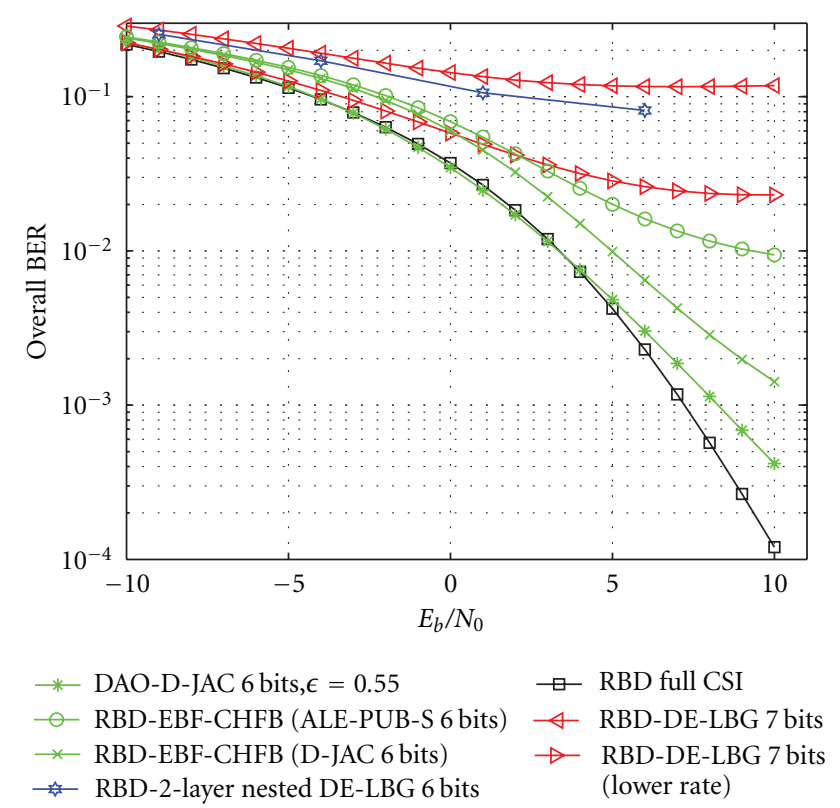

FIGURE 2: BER performance comparison for 2 users and a single stream per user, at mobile speeds of $3 \mathrm{~km} / \mathrm{h}$. Each stream uses 16 QAM symbols, exception made in RBD-DE-LBG "lower rate", where QPSK symbols are used.

(MRC) power, constrained to updating only one vector element on each slot. In particular, the ALE-PUB-S operates sequentially (round-robin) on the elements of the $N_{t} N_{r}$ vectorized channel $\tilde{\mathbf{h}}_{i}$. For each slot, the power and phase of the updated element are uniformly quantized and fed back to the transmitter. For $n_{b}=6$, three bits are used for power and phase quantization, respectively. The associated simulation curves are labeled "RBD-EBF-CHFB (ALE-PUB-S 6 bits)" and the power and phase of the PU are quantized with three bits each.

Other recursive eigenbeamforming methods can also be employed, but have not been benchmarked. For example, in [18] the optimal beamformer is parametrized as angles corresponding to a cascade of Givens rotors and unitmodulus scalings, and each parameter is tracked with a single-bit structure similar to the adaptive delta modulation concept from early speech coding techniques [19]. However, as the number of real-valued parameters in the considered scenario is $2\left(N_{t} N_{r}-1\right)=14>n_{b} \in\{6,7\}$, it is not possible to update all the parameters every slot.

4.2. Bit Error Rate and Ergodic Capacity. The average BER simulations are shown in Figure 2. It can be seen that the DAO-D-JAC algorithm outperforms centralized alternatives operating at the same feedback rate. We note that nonrecursive DE-LBG suffers from error floors, which is consistent with $[3,4]$ because the codebook size does not scale with the SNR. On the other hand, the estimation of $\mathbf{Q}_{i}$ may produce a slight degradation on the performance of DAO-D-JAC. This is an implementation-dependent feature beyond the scope of this work. Furthermore, the estimation can be improved if the transmitter forwards the used transmit weights in a

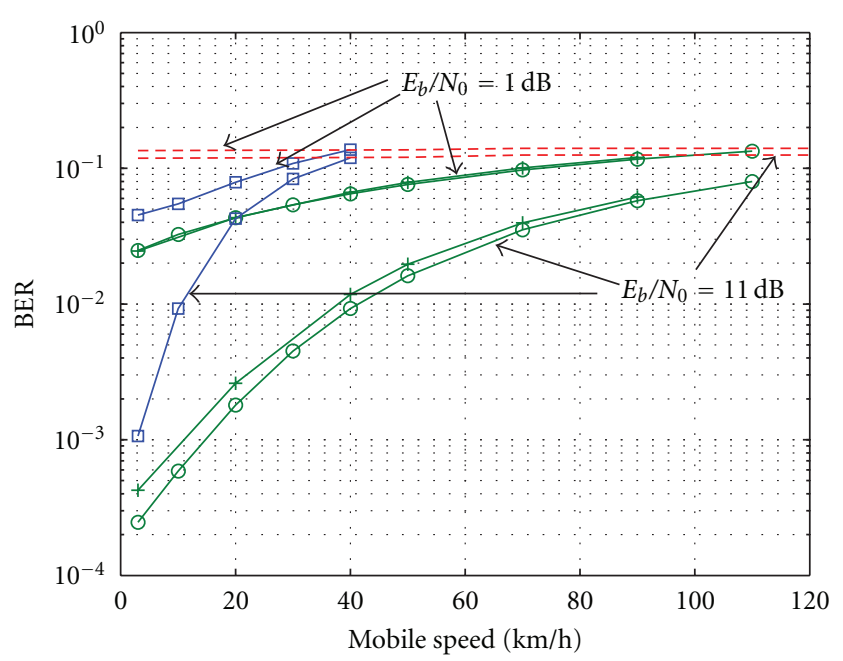

$\begin{array}{llll}+ & \text { DAO-D-JAC } 6 \text { bits, no filtering } & --- & \text { RBD-DE-LBG } 7 \text { bits } \\ \square & \text { RBD-EBF-CHFB (D-JAC } 6 \text { bits) } & - & \text { DAO-D-JAC } 6 \text { bits }\end{array}$

FIGURE 3: Impact of mobile speed on BER performance for the twouser, single stream per user scenario.

common control channel. It is also shown that the limitedfeedback degradation for RBD is less severe when smallersize constellations such as QPSK are used.

Figure 3 gives the degradation of the BER performance as function of the speed. Note that increasing the speed decreases the channel coherence time and thus limits the time available for convergence and tracking, even though the processing delay of the feedback message is neglected. It is shown that the centralized solution based on closedloop eigenbeamforming outperforms the nonrecursive DELBG up to mobile speeds of approximately $40 \mathrm{~km} / \mathrm{h}$, and that the proposed DAO-D-JAC degrades less than the recursive centralized solution, as the speed increases. It can be seen that the filtering parameter $\epsilon$ does not have a noticeable impact at low SNRs.

Figure 4 shows the average of the instantaneous total rate $\log _{2}\left(1+\rho_{1}\right)+\log _{2}\left(1+\rho_{2}\right)$, where the per-user SINRs $\rho_{i}$ are defined in (4). We note that the RBD-DE-LBG 7-bit codebook curves generally agree with Figure 2 of [4], and that a one stream per user strategy is preferable over the twostream alternative, when the nonrecursive 7-bit codebook is used. It is also shown that the two-layer nested DE-LBG with $n_{b}=6$ improves the performance, compared to the nonrecursive DE-LBG with $n_{b}=7$. However, additional layers would be required to further boost the throughput, as illustrated by the results for recursive channel feedback using ALE-PUB-S and D-JAC.

Finally, the performance of the angle forwarding mechanism as compensation for feedback errors is shown in Figure 5, for the slowly fading case. The simulations assume Gray encoding of the quantized rotor angles and i.i.d. feedback bit errors with a given probability. It can be seen that the compensation is effective, and allows the proposed 

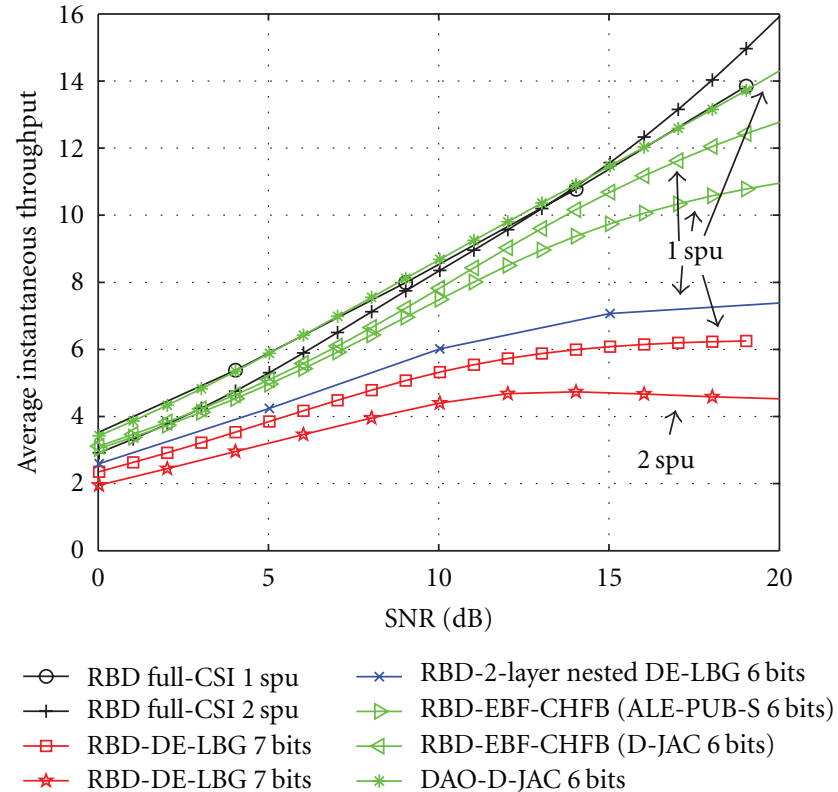

FIgURE 4: Average of total instantaneous rates at mobile speeds of $3 \mathrm{~km} / \mathrm{h}$ in the two-user case. "spu" refers to the number of streams per user.

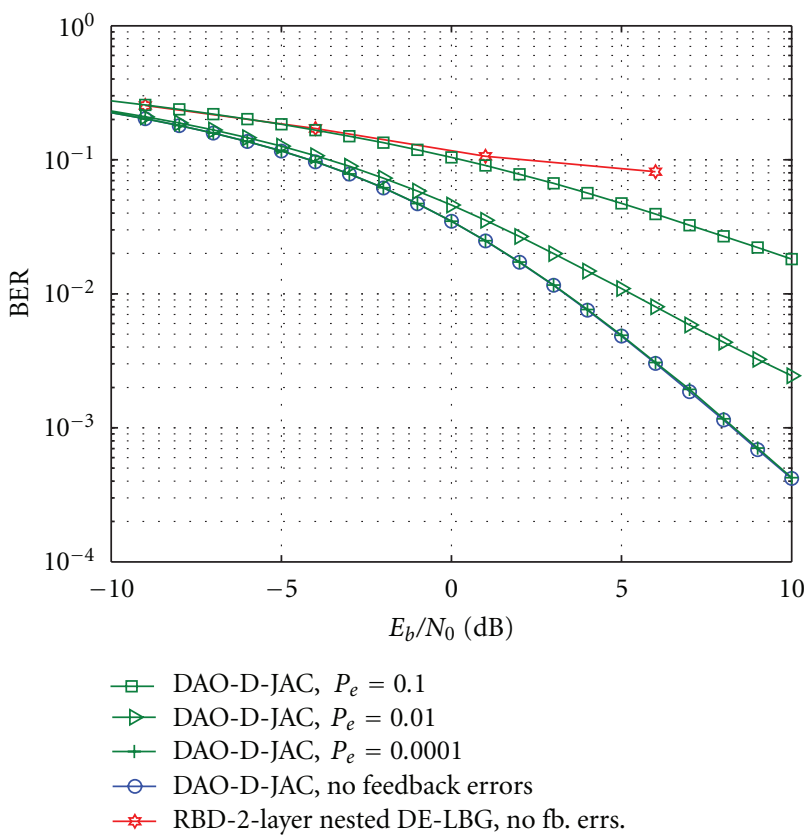

FIgure 5: Compensation of feedback errors with angle forwarding mechanism for the two-user system with mobile speeds of $3 \mathrm{~km} / \mathrm{h}$. $P_{e}$ refers to the probability of error in a feedback bit.

AOD-JAC to outperform the centralized alternative up to feedback bit error probabilities of $10 \%$. Furthermore, the per-user overhead incurred in the downlink path is small, namely $6 /(160 \cdot 4) \approx 0.9 \%$. The effect of the feedback errors under $1 \%$ probability is illustrated for static channels in Figures 6 and 7. This suggests that as long as no errors are found in the forwarded angles, reasonably low amounts of

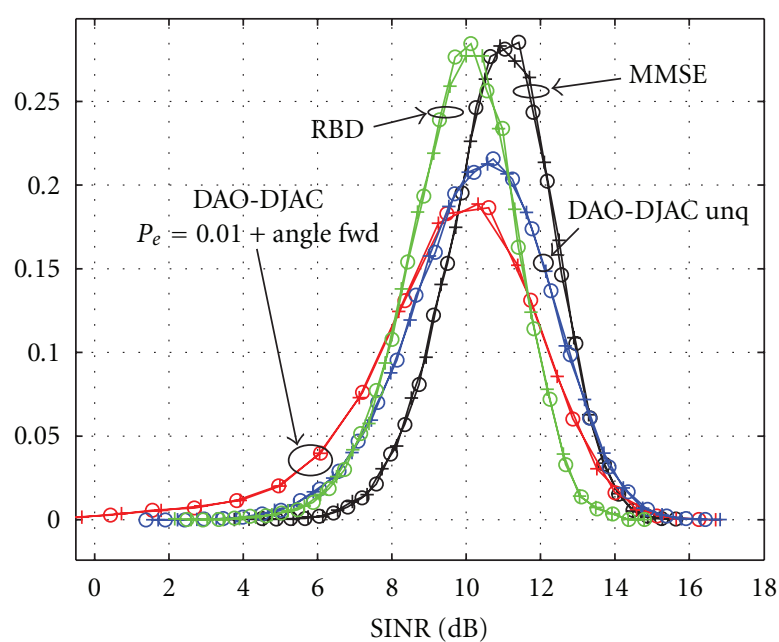

FIGURE 6: SINR comparison for two users under perfectly known static channels. "DAO-D-JAC unq" uses unquantized rotor angles for the update (5). "DAO-D-JAC + angle fwd" refers to the DAO technique, where the rotor angles are quantized with 3 bits each and feedback bit errors are compensated with the angle forwarding mechanism of Section 3.1.

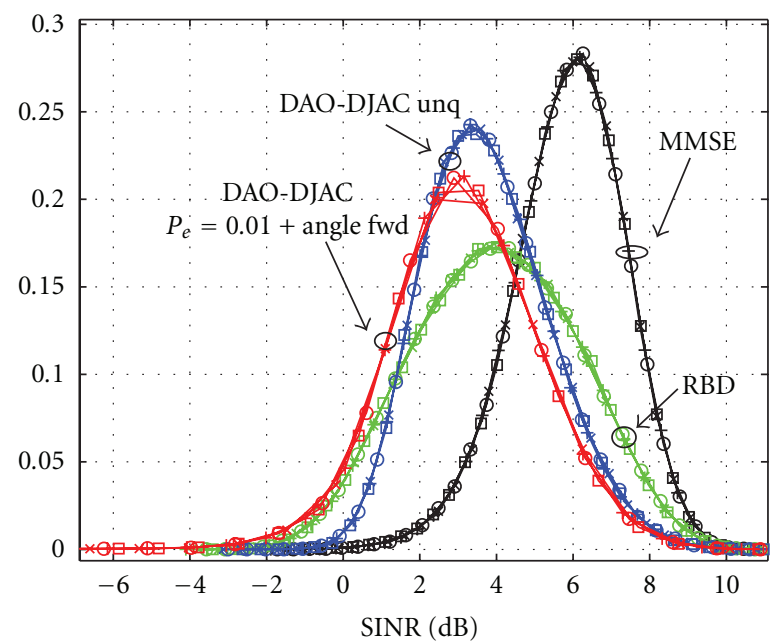

FIGURE 7: SINR comparison for four users under perfectly known static channels. "DAO-D-JAC unq" uses unquantized rotor angles for the update (5). "DAO-D-JAC + angle fwd" refers to the DAO technique, where the rotor angles are quantized with 3 bits each and feedback bit errors are compensated with the angle forwarding mechanism of Section 3.1.

feedback errors do not compromise the convergence of the DAO technique.

4.3. Convergence in Static Channels. In this section, we briefly explore the convergence properties of the DAO technique which underlies the proposed decentralized MUMIMO solution. The results presented in the previous section suggest that the DAO solutions are good for small systems. To further characterize the properties of the DAO method, we study the performance of the transmit weights 
obtained upon convergence in static channels, under fullCSI conditions. Note that even though the DAO is similar to the AO technique [9], we cannot trivially derive converge properties from existing $\mathrm{AO}$ results, and therefore rely on simulations with large number of channel realizations. All the DAO-D-JAC solutions have been obtained with a fixed number of iterations, where the precoders of the last step are used. We noticed some oscillations in the per-user SINR learning curves when increasing the probability of feedback errors.

Figures 6 and 7 give the distributions of the per-user SINRs obtained after convergence of the DAO weights, when one stream per user is transmitted and the ratio of transmit power to noise power is $N_{u} / \sigma^{2}=7 \mathrm{~dB}$. Figure 6 shows that the DAO solution for two users performs comparably to the RBD, but is suboptimal when compared to the MMSE solution in Section 4.1.2.

Figure 7 shows the simulation results when the number of users is increased to four. It can be seen that the DAO solution performs considerably worse than the MMSE solution, but it does not break down. This does not necessarily mean, however, that the limited-feedback DAO will perform worse than the centralized MMSE, since it is not known how the MMSE solution degrades when channel feedback mechanisms are used. A limited-feedback performance comparison for this scenario is deferred to further research. We also note that the RBD solution can be refined by means of the iterative RBD (IRBD) technique, as described in [1].

\section{Conclusion}

This paper presented a limited-feedback decentralized multiuser MIMO solution, tailored for the particular case of single data stream per user transmission and low mobility scenarios. The proposed technique exploits interference rejection combiner receivers to achieve a receiverbased MU-MIMO solution which avoids feeding back the channel matrix explicitly, where the transmitter adapts the transmit weights according to compact feedback messages sent by the mobile receivers. The weight recursion features low computational complexity and is based on complex-valued Givens rotors, where a minimum of one rotor per update can be used. The proposed transmission scheme is shown to obtain uniform average BER and throughput performance improvements, when compared with existing centralized MU-MIMO solutions using channel feedback methods operating at the same feedback rate. A feedback error compensation mechanism is presented, which enables the operation of the proposed algorithm under moderate probabilities of feedback bit errors.

\section{References}

[1] V. Stankovic and M. Haardt, "Generalized design of multi-user MIMO precoding matrices," IEEE Transactions on Wireless Communications, vol. 7, no. 3, pp. 953-961, 2008.
[2] K.-K. Wong, R. D. Murch, and K. B. Letaief, "A joint-channel diagonalization for multiuser MIMO antenna systems," IEEE Transactions on Wireless Communications, vol. 2, no. 4, pp. 773-786, 2003.

[3] N. Ravindran and N. Jindal, "Limited feedback-based block diagonalization for the MIMO broadcast channel," IEEE Journal on Selected Areas in Communications, vol. 26, no. 8, pp. 1473-1482, 2008.

[4] B. Song, F. Roemer, and M. Haardt, "Efficient channel quantization scheme for multi-user MIMO broadcast channels with RBD precoding," in Proceedings of the IEEE International Conference on Acoustics, Speech and Signal Processing (ICASSP '08), pp. 2389-2392, Las Vegas, Nev, USA, April 2008.

[5] J. H. Winters, "Optimum combining in digital mobile radio with cochannel interference," IEEE Transactions on Vehicular Technology, vol. 33, no. 3, pp. 144-155, 1984.

[6] E. Zacarías, S. Werner, and R. Wichman, "Distributed Jacobi eigen-beamforming for closed-loop MIMO systems," IEEE Communications Letters, vol. 10, no. 12, pp. 825-827, 2006.

[7] E. Zacarías, S. Werner, and R. Wichman, "Enhanced partial update beamforming for closed loop MIMO systems," in Proceedings of the IEEE International Symposium on Personal, Indoor and Mobile Radio Communications (PIMRC '06), Helsinki, Finland, September 2006.

[8] A. L. Anderson, J. R. Zeidler, and M. A. Jensen, "Reducedfeedback linear precoding with stable performance for the time-varying MIMO broadcast channel," IEEE Journal on Selected Areas in Communications, vol. 26, no. 8, pp. 14831493, 2008.

[9] J. C. Bezdek and R. J. Hathaway, "Some notes on alternating optimization," in Advances in Soft Computing-AFSS 2002, vol. 2275 of Lecture Notes In Computer Science, Springer, Berlin, Germany, 2002.

[10] G. H. Golub and C. F. Van Loan, Matrix Computations, The Johns Hopkins University Press, Baltimore, Md, USA, 2nd edition, 1989.

[11] 3GPP, "Evolved Universal Terrestrial Radio Access (EUTRA); Multiplexing and channel coding," 3rd Generation Partnership Project (3GPP), TS 36.212, September 2008, http://www.3gpp.org/ftp/Specs/html-info/36212.htm.

[12] B. Bandemer, M. Haardt, and S. Visuri, "Linear MMSE multiuser MIMO downlink precoding for users with multiple antennas," in Proceedings of the IEEE 17th International Symposium on Personal, Indoor and Mobile Radio Communications, (PIMRC '06), Helsinki, Finland, September 2006.

[13] Y. Linde, A. Buzo, and R. M. Gray, "An algorithm for vector quantizer design," IEEE Transactions on Communications Systems, vol. 28, no. 1, pp. 84-95, 1980.

[14] A. Narula, M. J. Lopez, M. D. Trott, and G. W. Wornell, "Efficient use of side information in multiple-antenna data transmission over fading channels," IEEE Journal on Selected Areas in Communications, vol. 16, no. 8, pp. 1423-1436, 1998.

[15] J. H. Kim, W. Zirwas, and M. Haardt, "Efficient feedback via subspace-based channel quantization for distributed cooperative antenna systems with temporally correlated channels," EURASIP Journal on Advances in Signal Processing, vol. 2008, Article ID 847296, 13 pages, 2008.

[16] B. Mondal and R. W. Heath Jr., "Channel adaptive quantization for limited feedback MIMO beamforming systems," IEEE Transactions on Signal Processing, vol. 54, no. 12, pp. 47174729, 2006. 
[17] V. Raghavan, R. W. Heath Jr., and A. M. Sayeed, "Systematic codebook designs for quantized beamforming in correlated MIMO channels," IEEE Journal on Selected Areas in Communications, vol. 25, no. 7, pp. 1298-1310, 2007.

[18] J. C. Roh and B. D. Rao, "Efficient feedback methods for MIMO channels based on parameterization," IEEE Transactions on Wireless Communications, vol. 6, no. 1, pp. 282-292, 2007.

[19] N. S. Jayant, "Digital coding of speech waveforms: PCM, DPCM, and DM quantizers," Proceedings of the IEEE, vol. 62, no. 5, pp. 611-632, 1974. 

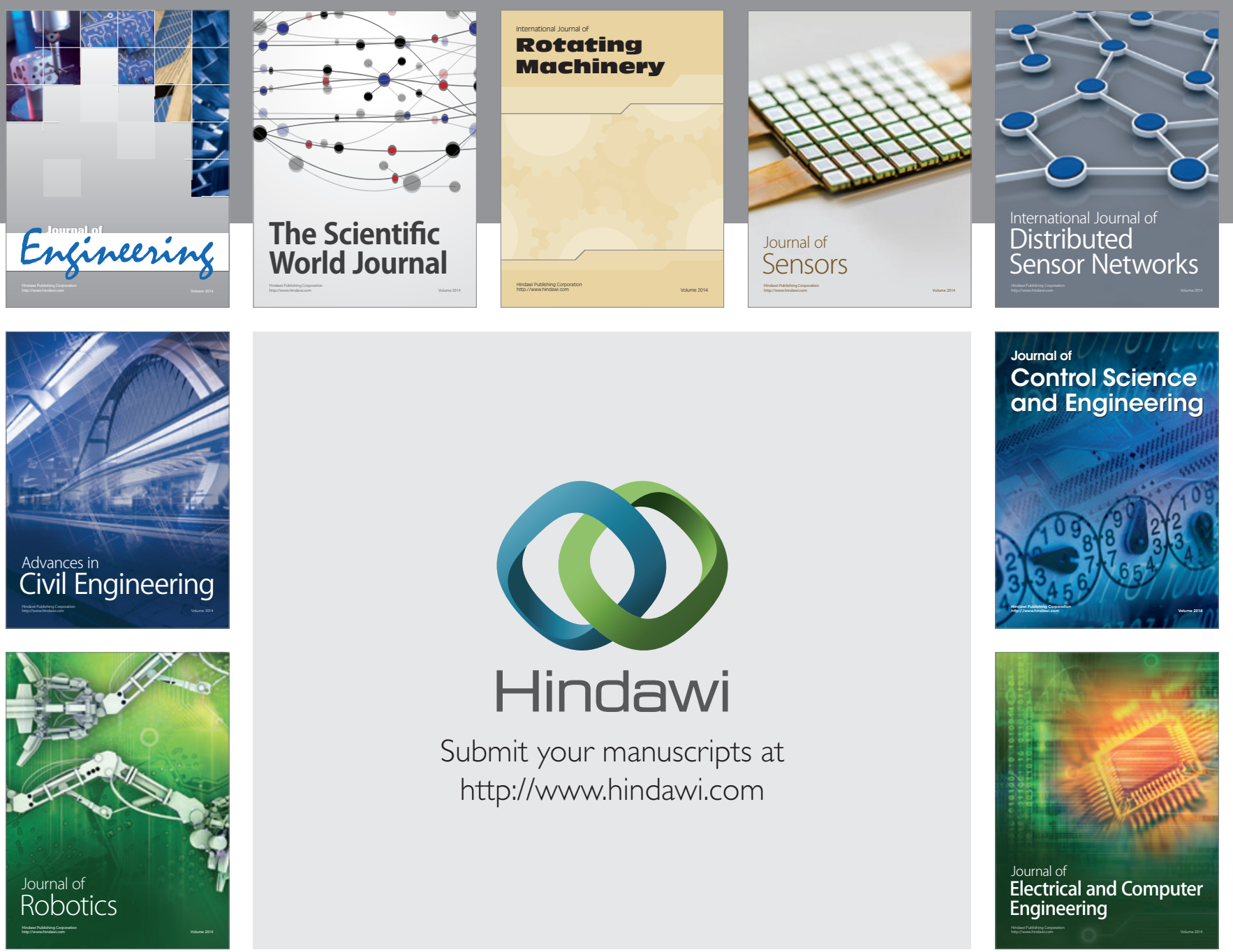

Submit your manuscripts at

http://www.hindawi.com
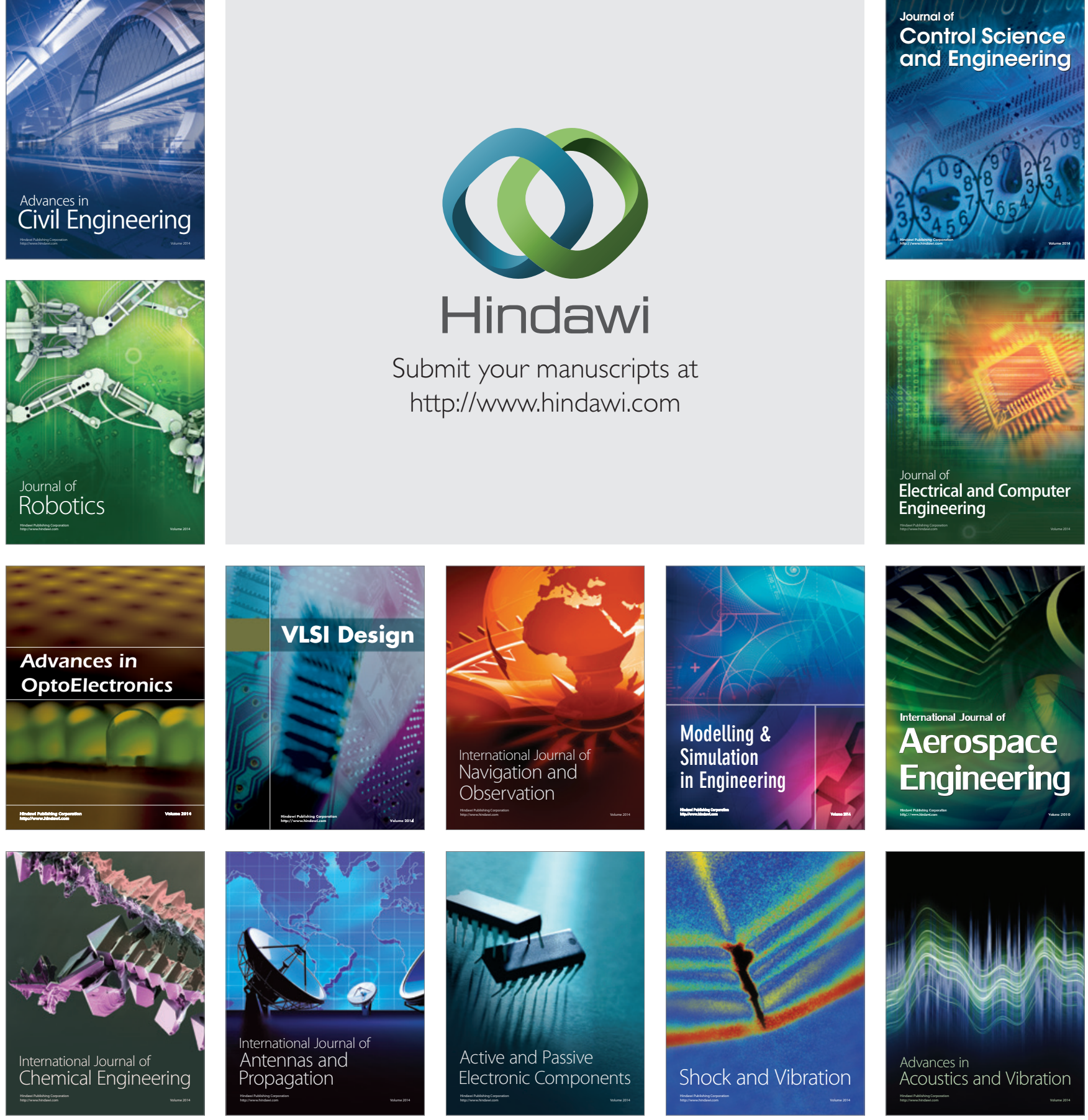